\title{
Hematoma cerebeloso como complicación de artrodesis lumbar. A propósito de un caso y revisión de la literatura
}

\author{
M. Castle; C. Barrena; N. Samprón; I. Arrese
}

Servicio de Neurocirugía. Hospital Universitario Donostia. San Sebastián. España.

\section{Resumen}

El hematoma cerebeloso (HCD) como complicación en cirugía de raquis, es menos frecuente que el hematoma a distancia secundario a la cirugía intracraneal. Se manifiesta principalmente con cefalea o deterioro del nivel de consciencia y se ha asociado a la pérdida de LCR intra o postoperatoria.

Presentamos un caso de HCD en una paciente de 55 años tras cirugía instrumentada de columna lumbar con fístula de LCR oculta, además de una revisión de la literatura con análisis de sus posibles causas, así como las implicaciones terapéuticas.

PALABRAS CLAVE. Hematoma cerebeloso a distancia. Cirugía de raquis. Fístula de LCR.

Remote cerebellar haemorrhage after lumbar arthrodesis: Case report and literature review

\section{Summary}

Remote cerebellar haemorrhage $(\mathrm{RCH})$ is a rare complication of spinal surgery, less frequent than the RCH observed after an intracranial surgery. The patients principally complained of headache or were presented with deterioration in mental status.

We report a case of $\mathrm{RCH}$ in a 55 years old woman that underwent lumbar arthrodesis with occult dural defect. We review the literature, analysing its causes and therapeutic implications.

KEY WORDS. Remote cerebellar haemorrhage. Spinal surgery. Cerebrospinal fluid leakage.

\section{Introducción}

Desde que en 1981, Chadduck $^{6}$ publicase el primer caso de hematoma cerebeloso a distancia (HCD) como

Recibido: 13-04-11. Aceptado: 7-07-11 complicación en cirugía de raquis, sólo han sido publicados 24 casos en total. Menos frecuente que el hematoma a distancia secundario a la cirugía intracraneal, presenta múltiples similitudes con esta entidad: se manifiesta con cefalea o deterioro del nivel de consciencia en un rango de tres horas a ocho días después de la cirugía ${ }^{10,17,18}$ y muestra un patrón característico en la TC craneal llamado el "signo de la cebra", que en la mayoría de los casos es hallado en ambos hemisferios cerebelosos.

Presentamos un caso de HCD tras cirugía instrumentada de columna lumbar, y revisamos la literatura, analizando las posibles causas, así como las implicaciones terapéuticas.

\section{Caso clínico}

Mujer de 55 años, sin antecedentes de interés, a quien se le realiza artrodesis circunferencial L4-L5 por vía posterior debido a un cuadro de inestabilidad lumbar con clínica dolorosa de tres años de evolución. La intervención se llevó a cabo en decúbito prono, colocando tornillos transpediculares e implantes intersomáticos posterolaterales. Intraoperatoriamente no se observó ningún desgarro dural, por lo que se dejó drenaje epidural.

A las 48 horas presentó cefalea occipital de gran intensidad y signos de irritación meníngea, por lo que se realizó TC craneal urgente, en la que se observó un hematoma en los surcos de la parte superior del hemisferio cerebeloso derecho que ejercía efecto de masa sobre IV ventrículo Figura 1.

La paciente fue ingresada en CMI con tendencia al sueño y el drenaje lumbar fue retirado. Tres horas más tarde, presentó disminución brusca del nivel de consciencia, interviniéndose de forma urgente mediante craniectomía suboccipital y evacuación del hematoma.

Ocho días más tarde la paciente pasó a planta con buen nivel de consciencia y signos de afectación cerebelosa, pero ingresó nuevamente en CMI tras cinco días en planta con diagnostico de meningitis por E. Coli, objetivándose en ese momento salida de LCR por la herida quirúrgica. 


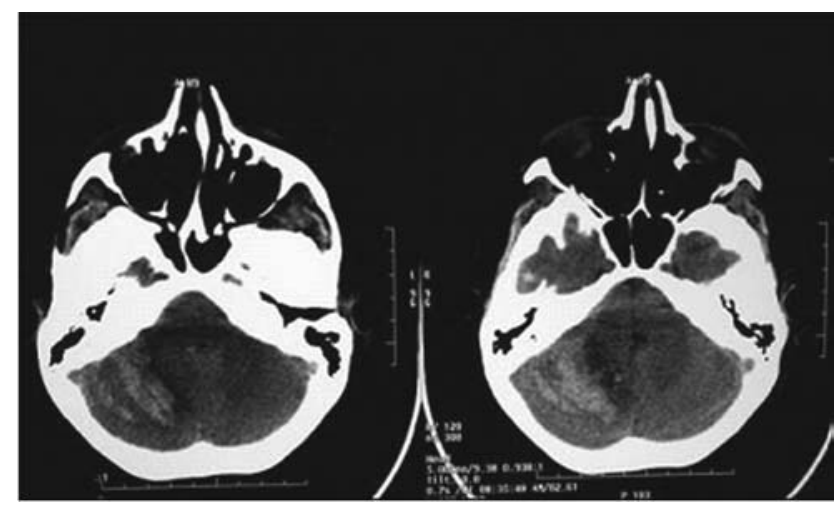

Figura 1. TC craneal sin contrastes, 48 horas después de la cirugía, en el que muestra un hematoma cerebeloso derecho con el" signo de la cebra" localizado en hemisferio cerebeloso derecho, con deformación secundaria del cuarto ventrículo.

La fístula de LCR lumbar se manejó de manera conservadora, recibiendo tratamiento antibiótico y reposo en cama.

Tras un ingreso hospitalario prolongado donde recibió tratamiento rehabilitador, la paciente realiza vida independiente y sólo persisten signos de afectación cerebelosa subclínicos. Se ha realizado Angio-RM craneal y cervical en la que no se ha encontrado patología vascular.

\section{Discusión}

Hasta la fecha sólo se han publicado 26 casos de HCD después de cirugía espinal, la mayoría mujeres y tras cirugía lumbar. Como se puede observar en la Tabla 1, en la que se resumen las características de los casos publicados, todos los pacientes sufrieron desgarro dural, excepto en dos en que no se hace referencia ${ }^{5}$, el cual se objetivo durante la intervención o se descubrió a posteriori. Esto convierte a la fístula de LCR en el factor causal fundamental para la aparición de estos hematomas. La hipotensión intracraneal provocada por la salida de LCR, con el consiguiente elongamiento y oclusión de las venas puente, generarían la aparición de un infarto venoso hemorrágico ${ }^{16}$. El tiempo de presentación tras la cirugía, el patrón radiográfico "en cebra" y la localización en la parte superior de los hemisferios cerebelosos descrito en todos los casos, incluido el nuestro, apoyan claramente esta teoría. Dos casos publicados como complicación de drenajes lumbares ${ }^{2}$ y el hallazgo de presión intracraneal baja durante la ventriculostomía en otro caso ${ }^{19}$ favorecen aún más la asunción de esta etiología como factor causal fundamental en la aparición de HCD. Por ello, consideramos que en los casos en los que se produzca HCD tras cirugía espinal, aunque el desgarro dural no sea objetivado durante la intervención, como sucedió en nuestro caso, el neurocirujano debe asumir que este evento se ha producido y actuar en consecuencia. Esto es especialmente importante ante la presencia de un drenaje epidural, el cual aumentará la salida de LCR con la consiguiente hipotensión. De los casos publicados, en 13 de ellos los pacientes tenían drenajes epidurales colocados.

A diferencia de los casos de HCD publicados tras cirugía intracraneal, en los casos tras cirugía de raquis no se ha descrito ningún otro factor determinante aparte de la apertura dural. Factores asociados al HCD en cirugía craneal como la hipertensión arterial, la anticoagulación o las coagulopatías no han sido relacionados. Así mismo, a diferencia de HCD secundarios a cirugía craneal, los casos descritos en intervenciones espinales parecen no asociarse a la colocación del paciente ${ }^{14}$. En cuanto a la presencia de malformaciones vasculares, sólo se encontró un caso con una malformación arterio-venosa en fosa posterior ${ }^{13}$. En nuestro caso, no encontramos ningún factor de riesgo de sangrado ni malformación subyacente.

Revisando la evolución de todos los casos publicados, el síntoma más frecuente es la cefalea en el 58\% de los pacientes, seguido de deterioro del nivel de consciencia 37\%. En el resto la disartria fue el síntoma inicial, observando la aparición de los síntomas desde las primeras tres horas tras la cirugía hasta los siete días posteriores. El tratamiento quirúrgico fue necesario en 8 pacientes por deterioro neurológico progresivo, con edema cerebeloso y obstrucción del IV ventrículo en las TC de control. El 58\% no presentó déficit neurológico al alta y el $12,5 \%$ de los pacientes falleció. Con todo ello, pese a que en la mayoría de los casos el HCD pueda ser manejado de forma conservadora y tenga una evolución relativamente benigna, como se ilustra en nuestro caso, el paciente debe ser estrechamente vigilado pues las posibilidades de deterioro grave no son desdeñables.

\section{Conclusiones}

Ante un paciente intervenido de cirugía raquídea que presenta cefalea intensa o deterioro neurológico, la existencia de un HCD siempre debe ser sospechada, por lo que si existe drenaje epidural este debe ser retirado y se realizará una TC craneal. Si la TC craneal muestra un patrón "en cebra" se debe asumir la existencia de una fístula de LCR aunque durante la cirugía no se haya apreciado apertura dural. Inicialmente el paciente puede ser manejado de manera conservadora, pero es obligada una vigilancia estricta ante la posibilidad de deterioro neurológico y necesidad de evacuación quirúrgica. 


\begin{tabular}{|c|c|c|c|c|c|c|c|c|c|c|}
\hline 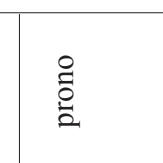 & & 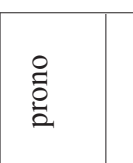 & & $\frac{a}{2}$ & 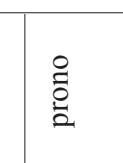 & 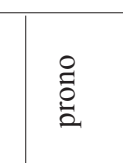 & & & & 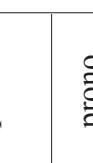 \\
\hline 意音 & $\frac{5}{1}$ & 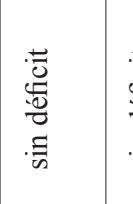 & 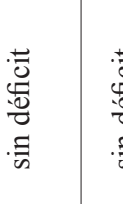 & & $\frac{1}{2}$ & 亳 & & & & \\
\hline$=$ & 言 & $=$ & - & $=\cong$ & & . & $=$ & & & \\
\hline$\overline{ }$ & $\frac{\vdots}{\vdots}$ & $\overline{=}$ & $=$ & $=$ & & . & $\bar{z}$ & & 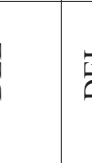 & \\
\hline 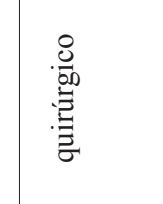 & & 量 & & 量量 & & 喜 & 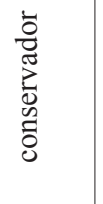 & & & \\
\hline & & & & & & & 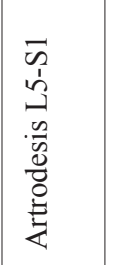 & & & \\
\hline 芭 & $\frac{\mathscr{e}}{\cong}$ & : & 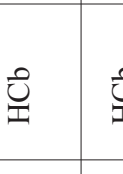 & 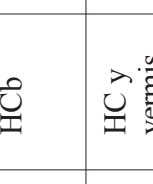 & $\frac{b}{x}$ & 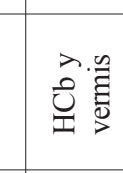 & $\frac{\hat{t}}{\underline{x}}$ & ع & : & \\
\hline ‡ & $\frac{\mathscr{L}}{\mathrm{g}}$ & $=$ & $m=$ & $\neq \approx$ & $\approx$ & $\underline{\underline{g}}$ & $\therefore$ & & 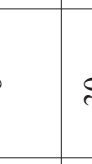 & \\
\hline 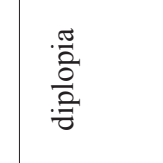 & $\frac{\frac{2}{3}}{3}$ & 童 & $\frac{\underline{a}}{\bar{z}}$ & & $\frac{1}{5}$ & 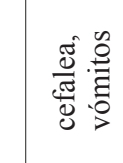 & $\frac{1}{\bar{z}}$ & 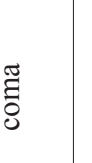 & & 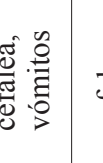 \\
\hline z & $F$ & $=$ & $=0$ & $\overline{\bar{n}} \overline{\bar{\sigma}}$ & $z$ & $\approx$ & $\bar{n}$ & s. & $:$ & \\
\hline $\bar{z}$ & $\bar{z}$ & $\bar{z}$ & $\bar{z}=$ & $=7=$ & $\bar{z}$ & $=$ & $\Sigma$ & & ; & \\
\hline 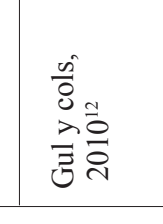 & & & & & & 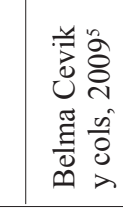 & & & & \\
\hline
\end{tabular}




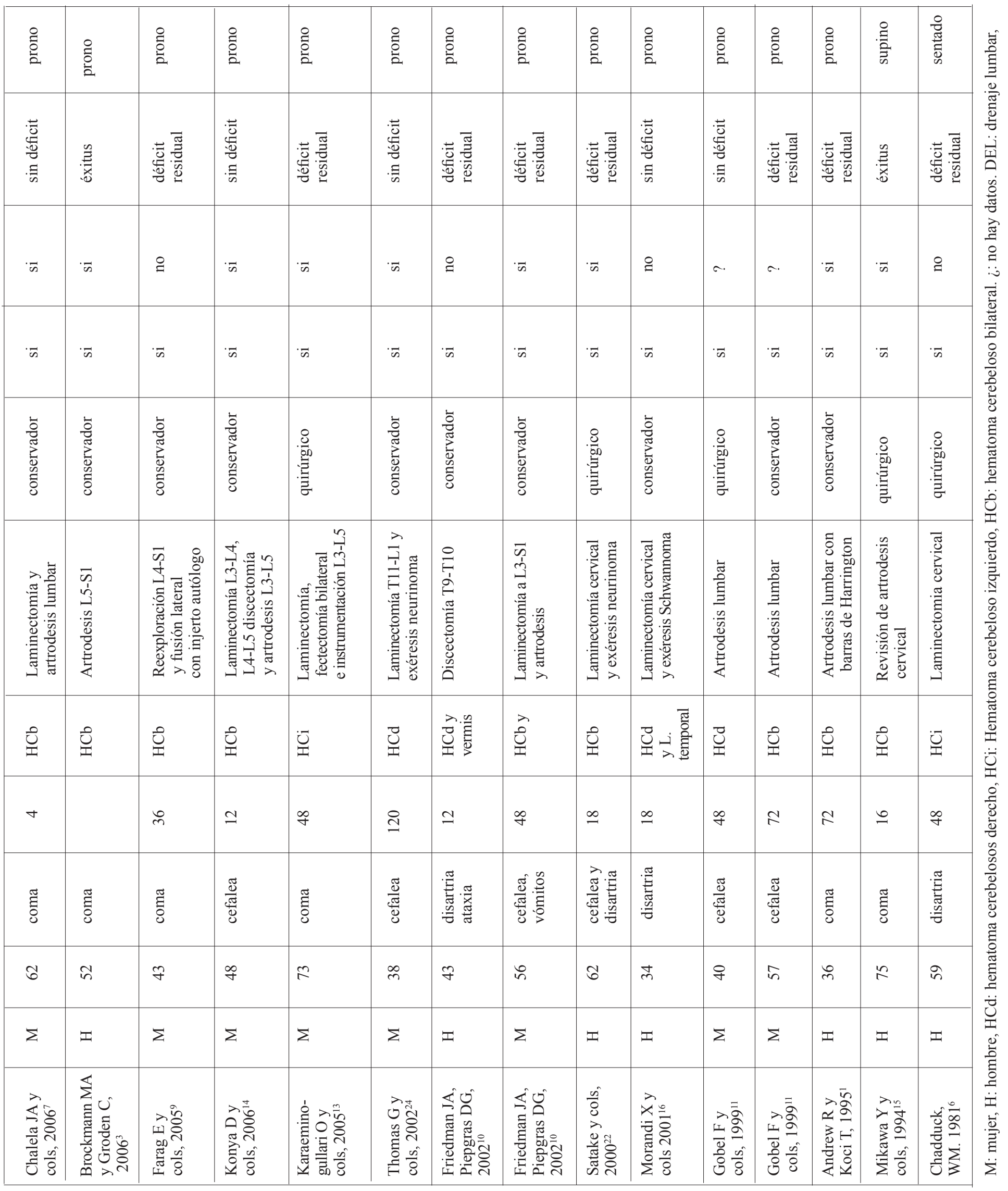




\section{Bibliografía}

1. Adrews, R.T., Koci, T.M.: Cerebellar herniation as a complication of an occult postoperative lumbar dural defect. Am J Neuroradiol 1995; 16: 1312-1315.

2. Bernal-Gracia, L.M., Cabezudo-Artero, J.M., OrtegaMartínez, M., et al.: Hematoma de cerebelo como complicación de drenaje lumbar. Presentación de dos casos y revisión de la literatura. Neurocirugía 2008; 19: 440-445.

3. Brockmann, M.A., Nowak, G., Reusche, E., Russlies, M., Petersen, D.: Zebra sign: cerebellar bleeding characteristic of cerebrospinal fluid loss. J Neurosurg 2005; 102: 1159-1162.

4. Calisaneller, T., Yilmaz, C., Ozger, O., Caner, H.: Remore cerebellar haemorrhage alter spinal surgery. The Canadian Journal of Neurological Science 2007; 34: 483-484.

5. Cevik, B., Kirbas, I., Cakir, B., Akin, K., Teksam, M.: Remore cerebellar hemorrahage after lumbar spinal sugery. European Journal of Rdiology 2009; 70: 7-9.

6. Chadduck, W.M.: Cerebellar hemorrhage complicating cervical laminectomy. Neurosurgery 1981; 9:185-189.

7. Chalela, J.A., Monroe, T., Kelley, M., et al.: Cerebellar haemorrhage caused by remote neurological surgery. Neurocrit Care 2006; 5: 30-34.

8. Enel, D., Blamoutier, A., Bacon, P., Gentili, M.E.: Spine surgery associeated with fatal cerebellar haemorrhage. European Journal of Anaesthesiology 2009; 26: 891-892.

9. Farag, E., Abdou, A., Riad, I., Borsellino, A.R., Scubert, A.: Cerebellar Hemorrhage caused by cerebrospinal fluid leak after spine surgery. Anesth Analg 2005; 100: 545-546.

10. Friedman, J.A., Ecker, R.D., Piepgras, D.G., Duke, D.A.: Cerebellar hemorrhage alter spinal surgery: Report of two cases and literature review. Neurosurgery 2002; 50: 13611364.

11. Göbel, F., Heidecke, V., Hube, R., Reichel, H., Held, A., Hein, W.: Cerebellar haemorrhage as an early complication of apinal operations. Two cases reports and review of the literature. Z Orthop Ihere Grenzgeb 1999; 137: 371-375.

12. Gul, S., Kalacy, M., Acikgoz, B.: A Rare Complication of Spinal Surgery: Cerebelllar Hemorrhage. Turkish Neurosurgery 2010; 20: 413-417.

13. Karaeminogullari, O., Ataly, B., Sahin, O. et al.: Remote Cerebellar hemorrhage after spinal surgery complicated by dural tear: case report and literatura review. Neurosurgery 2005; 57: ONS-215.

14. Konya, D., Ozgen, S., Pmair, M.N.: Cerebellar hemorrhage after spinal surgery: case report and review of the literature. Eur Spine J 2006; 15: 95-99.
15. Mikawa, Y., Watanabe, R., Hino, Y., Ishii, R., Hirano, K.: Cerebellar haemorrhage complicating cervical durotomy and revision C1-C2 fusion. Spine 1994; 19: 1169-1171.

16. Morandi, X., Haegelen, C., Henaux, P.L., Riffaud, L.: Brain shift is central to the pathogenesis of intracerebral haemorrhage remote from the site of the initial neurosurgical procedure. Medical Hypotheses 2006; 67: 856-859.

17. Morofuji, Y., Tsunoda, K., Hiu, T., et al.: Remote cerebellar hemorrhage after cervical spinal suergery: two case reports and literature review. No Shinkei Geka 2009; 37: 11171122 .

18. Morofuji, Y., Tsunoda, K., Takeshita, T. et al.: Remote cerebellar hemorrhage following thoracic spinal surgery. Neurol Med Chir 2009; 49: 117-119.

19. Pallud, P., Belaïd, H., Aldea, S.: Succesfull management of a life threatening cerebellar haemorrhage following spine surgery, a case report. Asian Spine Journa 2009; 3, 3234.

20. Riera, M., Bergada, J.: Hemorragia cerebelosa tras cirugía espinal. Med Intensiva 2008; 32: 54-57.

21. Sasani, M., Sasani, H., Oze, A.F.: Bilateral Late Remote Cerebellar Hemorrhage as a Complication of a Lumbo-Peritoneal Shunt Applied After Spinal Arteriovenous Malformation Surgery. The Journal of Spinal Cord Medicine 2010; 33: 77-79.

22. Satake, K., Matsuyama, Y., Iwata, H., Sato, K., Kawakami, N.: Cerebellar haemorrhage complicating resection of a cervical intramedullary tumour. Spinal Cord 2000; 38: 504 .

23. Taek Kyum Nam, Seung Won Park, Byung Kook Min, Sung Nam Hwang: Remote Cerebellar Hemorrhage after lumbar Spinal Surgery. J Korean Neurosurg Soc 2009; 46: 501-509.

24. Thomas, G., Jayaram, H., Cudlip, S., Powell, M.: Supratentorial intraparenchymal haemorrhage secondary to intracranial CSF hypotension following spinal surgery. Spine 2002; 27: E410-E412.

Castle, M.; Barrena, C.; Samprón, N.; Arrese, I.: Hematoma cerebeloso como complicación de artrodesis lumbar. A propósito de un caso y revisión de la literatura. Neurocirugía 2011; 22: 574-578.

Correspondencia: María Castle. Servicio de Neurocirugía. Hospital Donostia. Paseo Dr. Beguiristain 109. 20014 San Sebastian. Guipuzcoa. España

Email: lumacara@yahoo.com 